(C) 2020 IEEE. Personal use of this material is permitted. Permission from IEEE must be obtained for all other uses, in any current or future media, including reprinting/republishing this material for advertising or promotional purposes, creating new collective works, for resale or redistribution to servers or lists, or reuse of any copyrighted component of this work in other works. 


\title{
Minimal time delivery of multiple robots
}

\author{
Miguel Aguiar ${ }^{1}$, Jorge Estrela da Silva ${ }^{2}$ and João Borges de Sousa ${ }^{1}$
}

\begin{abstract}
Consider a set of autonomous vehicles, each one with a preassigned task to start at a given region. Due to energy constraints, and in order to minimize the overall task completion time, these vehicles are deployed from a faster carrier vehicle.

This paper develops a dynamic programming (DP) based solution for the problem of finding the optimal deployment location and time for each vehicle, and for a given sequence of deployments, so that the global mission duration is minimal. The problem is specialized for ocean-going vehicles operating under time-varying currents. The solution approach involves solving a sequence of optimal stopping problems that are transformed into a set variational inequalities through the application of the dynamic programming principle (DPP). The optimal trajectory for the carrier and the optimal deployment location and time for each vehicle to be deployed are obtained in feedback-form from the numerical solution of the variational inequalities. The solution is computed with our open source parallel implementation of the fast sweeping method. The approach is illustrated with two numerical examples.
\end{abstract}

\section{INTRODUCTION}

Advances in sensor, computer, communication and navigation technologies, as well as in energy storage and composite materials, have enabled impressive developments in field robotics. However, this is just the beginning. In fact, up until recently, the focus of most deployments has been on single vehicle operations, while work on multi-vehicle control has mainly targeted problems in formation control.

Future robotic operations in remote and communicationschallenged areas will entail new aspects of cooperation among heterogeneous multi-domain vehicles. Some of these new aspects of cooperation are still being imagined today. Examples include mobile computing (mobility of software) and mobile computation (mobility of hardware), as well as distributed processing of data streams coming from different sensors. Other aspects are better understood. For example, one generic motion pattern for multi-domain vehicles concerns iterated rendezvous operations, in which vehicles exchange commands and data to decide where and when the next rendezvous takes place [7]. This motion pattern encompasses a significant number of complex motion-planning problems, including, for example, re-fueling, marsupial transportation [5] and cooperative pick-and-place [2]. Most of these problems also involve complex operational constraints such as dynamic obstacles, time-varying winds or water currents, deadlines, etc. The complexity of these problems comes from dynamic motion models, combinatorial explosion, dynamic constraints,

\footnotetext{
${ }^{1}$ M. Aguiar and J. Borges de Sousa are with LSTS, University of Porto, Porto, Portugal. E-mail: $\{\mathrm{m} . \mathrm{ag}$, jtasso $\}$ fe.up.pt

${ }^{2}$ J. Estrela da Silva is with ISEP, Instituto Politécnico do Porto, Porto, Portugal. E-mail: jes@isep.ipp.pt
}

large areas of operation (in space and time) and stagedependent cost functions. The combination of these difficulties makes it very difficult to derive a framework within which these problems can be formally formulated, analysed and solved. This is in part because of the hybrid nature of the statecontrol spaces in which these systems evolve. While vehicles evolve in continuous state-spaces, rendezvous activities signal transitions between discrete modes of operation and cost functions.

Here we present an approach to solve a class of multivehicle planning problems. In this class of problems, one ship is tasked to deliver $n$ Autonomous Underwater Vehicles (AUV) to $n$ different departure areas. The AUVs are then tasked to execute $n$ different tasks. The ship travels much faster than the AUVs, which are fuel constrained. All of these vehicles are subject to time-varying currents. The approach builds on DP methods applied to a hybrid-state model and on an efficient solver [1] of the Hamilton-Jacobi-Bellman partial differential equation resulting from the application of the DPP to this model.

The paper is organized as follows. Section II presents the formulation of the problem and section III discusses related work. Section IV describes the approach, including some results about it. Two numerical examples are presented in section V. The last section discusses the conclusions and future work.

\section{FORMULATION}

Consider a carrier vehicle which can transport and deploy $n$ smaller AUVs. We represent the position of the carrier vehicle by a point $\mathbf{x}=\left(x_{1}, x_{2}\right) \in \mathbf{R}^{2}$. The carrier is deployed at some point $\mathbf{x}^{0}$ at some time $t^{0}$, and the AUVs will be deployed in the order by which they are numbered at positions $\mathrm{x}^{i}$ and times $t^{i}, i=1, \ldots, n$. Once it is deployed, each AUV will perform some task, and we assume that we can compute a function $\theta^{i}: \mathbf{R} \times \mathbf{R}^{2} \rightarrow \mathbf{R}_{\geq 0}$ such that $\theta^{i}(t, \mathbf{x})$ equals the amount of time that AUV $i$ will take to complete its task if it is deployed at position $\mathbf{x}$ at time $t$.

The carrier is assumed to move according to the dynamics

$$
\dot{\mathbf{x}}(t)=\boldsymbol{u}(t)+\boldsymbol{v}(t, \mathbf{x}(t))
$$

where $\boldsymbol{u}$ is a control function satisfying $\|\boldsymbol{u}(t)\| \leq r$ (here and throughout, $\|\cdot\|$ denotes the Euclidean norm in $\mathbf{R}^{n}$ ), and $\boldsymbol{v}$ is a vector field modeling the water velocity. It is assumed that $\boldsymbol{v}$ is globally bounded, and that for any $(t, \mathbf{x})$ and $\mathbf{y}$ there is a trajectory $\boldsymbol{\xi}$ of (1) such that $\boldsymbol{\xi}(t)=\mathbf{x}$ and $\boldsymbol{\xi}\left(t^{\prime}\right)=\mathbf{y}$ for some $t^{\prime} \geq t$, even if $\|\boldsymbol{v}\|>r$ may happen occasionally.

We define $\Delta(s, \mathbf{y}, t, \mathbf{x})=s-t$ if $s \geq t$ and there is a trajectory $\boldsymbol{\xi}$ of (1) satisfying $\boldsymbol{\xi}(t)=\mathbf{x}$ and $\boldsymbol{\xi}(s)=\mathbf{y}$, and 
$\Delta(s, \mathbf{y}, t, \mathbf{x})=+\infty$ otherwise. The total mission time of AUV $i$ is defined as

$$
\mathcal{T}^{i}=\theta^{i}\left(t^{i}, \mathbf{x}^{i}\right)+\sum_{1 \leq k \leq i} \Delta\left(t^{k}, \mathbf{x}^{k}, t^{k-1}, \mathbf{x}^{k-1}\right),
$$

i.e., the amount of time that AUV $i$ spends in the carrier vehicle plus the amount of time it takes to complete its task.

The global mission duration is defined as

$$
\mathcal{T}=\max _{1 \leq i \leq n} \mathcal{T}^{i}
$$

Note that $\mathcal{T}$ is a function of all the deployment times and locations $\left(t^{i}, \mathbf{x}^{i}\right), i=0, \ldots, n$.

Problem 1: Given the deployment position $\mathrm{x}^{0}$ and time $t^{0}$ of the carrier vehicle, find deployment positions $\mathrm{x}^{i}$ and times $t^{i}$ for the AUVs that minimize $\mathcal{T}$.

The solution to Problem 1 is not necessarily unique. Here, we present a method which computes one of the solutions.

\section{RELATED WORK}

Dynamic programming methods have been extensively applied to solving optimal hybrid control problems [6, 12, 11]. The generic formulation addresses hybrid optimal control problems for systems where autonomous and controlled state jumps are allowed at the switching instants and the cost function includes running costs, as well switching costs between discrete states. The application of DP methods to these problems gives rise to a set of variational inequalities that typically do not have a closed-form solution. Relationships between adjoint processes in the Minimum Principle and the gradient of the value function in DP have also been studied in (e.g., in [11]).

These advances in hybrid systems research motivated the development of solutions to problems in multi-vehicle planning and execution control. For example, the problem of optimal coordinated path planning for two vehicles in which the path cost for one vehicle is a discontinuous function of the distance to the other vehicle is formulated and solved in [4]. The problem is solved with the help of three value functions. One is defined in the space-state of one vehicle and the other two are defined in the state-space of the other vehicle. Alton and Mitchell formulated and solved a problem of sequential coordinated pick and place for multiple robotic arms using DP methods and an implementation of the fast marching method [2]. The pick and place points are also the rendezvous locations of the robotic arms involved in the operation. Again, the solution of this iterated rendezvous problem is determined from several value functions, each one defined in the state-space of each robotic arm. Observe that the reduction of dimension comes from the modularity of the optimization problem. Space limitations preclude a thorough discussion of related work.

The contributions of this paper are as follows. First, a new formulation of a multi-stage multi-vehicle problem is introduced and addressed in the framework of sequential optimal stopping problems. The approach also encompasses more general vehicle task specifications. Each task is abstracted by a function returning the time to execute the task starting from a given time-position pair. This enables decoupled task optimization. Second, the approach deals with perturbations in the form of ocean currents that may overcome the motion capabilities of the vehicles for some periods of time. The assumption is that this does not preclude reachability of target positions. Third, an efficient numerical solver for the Hamilton-Jacobi-Bellman equation is used in a modular fashion to solve the sequential optimization problem. Finally, the cost function is a positional functional [10] (i.e, satisfies a non-decreasing property with respect to some arguments), thus making it possible to apply the principle of optimality.

\section{APPROACH}

\section{A. Dynamic Programming}

Our approach is to decompose the problem into a sequence of optimal stopping problems. Once we have such a decomposition, DP is used to solve these subproblems. The optimal deployment positions and times are then recovered from the corresponding value functions.

We start by defining the quantities $\hat{\mathcal{T}}^{i}, i=1, \ldots, n$, as

$$
\begin{aligned}
\hat{\mathcal{T}}^{n} & =\Delta\left(t^{n}, \mathbf{x}^{n}, t^{n-1}, \mathbf{x}^{n-1}\right)+\theta^{n}\left(t^{n}, \mathbf{x}^{n}\right) \\
\hat{\mathcal{T}}^{i} & =\Delta\left(t^{i}, \mathbf{x}^{i}, t^{i-1}, \mathbf{x}^{i-1}\right)+\max \left\{\hat{\mathcal{T}}^{i+1}, \theta^{i}\left(t^{i}, \mathbf{x}^{i}\right)\right\} .
\end{aligned}
$$

Each $\hat{\mathcal{T}}^{i}$ is a function of $\left(t^{k}, \mathbf{x}^{k}\right)$ for $k \geq i-1$, and its value is equal to the maximum mission time of AUV $i$, where these times are measured starting from $t^{i-1}$ (this is rigorously shown in the proof of Lemma 1$)$. We set $\hat{\mathcal{T}}^{n+1} \equiv 0$ so that relation (2) holds for $i=n$ also.

Lemma 1: For any choice of deployment times and locations, $\hat{\mathcal{T}}^{1}=\mathcal{T}$.

Proof: If $\Delta\left(t^{i}, \mathbf{x}^{i}, t^{i-1}, \mathbf{x}^{i-1}\right)$ is infinite for some $i$, then $\mathcal{T}$ and $\hat{\mathcal{T}}^{1}$ must both be infinite. Thus we henceforth assume that $\Delta\left(t^{i}, \mathbf{x}^{i}, t^{i-1}, \mathbf{x}^{i-1}\right)=t^{i}-t^{i-1}$ for all $i$. It follows that

$$
\mathcal{T}^{i}=\theta^{i}\left(t^{i}, \mathbf{x}^{i}\right)+t^{i}-t^{0} .
$$

Clearly we have $\hat{\mathcal{T}}^{n}=\mathcal{T}^{n}-\left(t^{n-1}-t^{0}\right)$, so that the relation

$$
\hat{\mathcal{T}}^{k}=\max _{j \geq k} \mathcal{T}^{j}-\left(t^{k-1}-t^{0}\right)
$$

holds for $k=n$. If it holds for $k=i+1$, then

$$
\begin{aligned}
\hat{\mathcal{T}}^{i} & =\max \left\{\max _{j \geq i+1} \mathcal{T}^{j}-\left(t^{i}-t^{0}\right), \theta^{i}\left(t^{i}, \mathbf{x}^{i}\right)\right\}+t^{i}-t^{i-1} \\
& =\max \left\{\max _{j \geq i+1} \mathcal{T}^{j}, \theta^{i}\left(t^{i}, \mathbf{x}^{i}\right)+t^{i}-t^{0}\right\}-\left(t^{i-1}-t^{0}\right) \\
& =\max _{j \geq i} \mathcal{T}^{j}-\left(t^{i-1}-t^{0}\right) .
\end{aligned}
$$

so by induction it holds for $k=1$, which proves the lemma.

We define the value function of AUV $i, V^{i}$ as

$$
\begin{aligned}
V^{i}\left(t^{i-1}, \mathbf{x}^{i-1}\right)=\inf _{t^{n}, \mathbf{x}^{n}, \ldots, t^{i}, \mathbf{x}^{i}} \hat{\mathcal{T}}^{i} & \\
=\inf _{t^{n}, \mathbf{x}^{n}, \ldots, t^{i}, \mathbf{x}^{i}} & \left\{\Delta\left(t^{i}, \mathbf{x}^{i}, t^{i-1}, \mathbf{x}^{i-1}\right)\right. \\
& \left.+\max \left\{\hat{\mathcal{T}}^{i+1}, \theta^{i}\left(t^{i}, \mathbf{x}^{i}\right)\right\}\right\} .
\end{aligned}
$$


The value of $V^{i}(t, \mathbf{x})$ equals the optimal value of the remaining mission time when vehicle $i-1$ is deployed at position $\mathbf{x}$ at time $t$. In particular, $V^{1}(t, \mathbf{x})$ equals the optimal value of $\mathcal{T}$ when $t^{0}=t$ and $\mathbf{x}^{0}=\mathbf{x}$. We define

$$
\mathcal{K}^{i}(t, \mathbf{x})=\max \left\{V^{i+1}(t, \mathbf{x}), \theta^{i}(t, \mathbf{x})\right\}
$$

to simplify the notation, where $V^{n+1} \equiv 0$. The following relation is easily derived:

$V^{i}\left(t^{i-1}, \mathbf{x}^{i-1}\right)=\inf _{t^{i}, \mathbf{x}^{i}}\left\{\Delta\left(t^{i}, \mathbf{x}^{i}, t^{i-1}, \mathbf{x}^{i-1}\right)+\mathcal{K}^{i}\left(t^{i}, \mathbf{x}^{i}\right)\right\}$.

This implies that we can determine the functions $V^{i}$ starting from $V^{n}$, which depends only on known data, namely $\theta^{n}$.

Theorem 1: Fix $t^{0}=\hat{t}^{0}$ and $\mathbf{x}^{0}=\hat{\mathbf{x}}^{0}$. Suppose that there exist $\left(\hat{t}^{i}, \hat{\mathbf{x}}^{i}\right), i=1, \ldots, n$ satisfying

$$
V^{i}\left(\hat{t}^{i-1}, \hat{\mathbf{x}}^{i-1}\right)=\Delta\left(\hat{t}^{i}, \hat{\mathbf{x}}^{i}, \hat{t}^{i-1}, \hat{\mathbf{x}}^{i-1}\right)+\mathcal{K}^{i}\left(\hat{t}^{i}, \hat{\mathbf{x}}^{i}\right) .
$$

Then $\left(\hat{t}^{1}, \hat{\mathbf{x}}^{1}\right), \ldots,\left(\hat{t}^{n}, \hat{\mathbf{x}}^{n}\right)$ is an optimal solution to Problem 1.

Proof: The relation

$$
\hat{\mathcal{T}}^{k}\left(\hat{t}^{n}, \hat{\mathbf{x}}^{n}, \ldots, \hat{t}^{k-1}, \hat{\mathbf{x}}^{k-1}\right)=V^{k}\left(\hat{t}^{k-1}, \hat{\mathbf{x}}^{k-1}\right)
$$

clearly holds for $k=n$. If it holds for $k=i+1$, then

$$
\begin{aligned}
\hat{\mathcal{T}}^{i} & =\Delta\left(\hat{t}^{i}, \hat{\mathbf{x}}^{i}, \hat{t}^{i-1}, \hat{\mathbf{x}}^{i-1}\right)+\max \left\{\theta^{i}\left(\hat{t}^{i}, \hat{\mathbf{x}}^{i}\right), \hat{\mathcal{T}}^{i+1}\right\} \\
& =\Delta\left(\hat{t}^{i}, \hat{\mathbf{x}}^{i}, \hat{t}^{i-1}, \hat{\mathbf{x}}^{i-1}\right)+\max \left\{\theta^{i}\left(\hat{t}^{i}, \hat{\mathbf{x}}^{i}\right), V^{i+1}\left(\hat{t}^{i}, \hat{\mathbf{x}}^{i}\right)\right\} \\
& =V^{i}\left(\hat{t}^{i}, \hat{\mathbf{x}}^{i}\right)
\end{aligned}
$$

In particular, $V^{1}=\hat{\mathcal{T}}^{1}=\mathcal{T}$, so $\left(\hat{t}^{i}, \hat{\mathbf{x}}^{i}\right)$ is optimal.

The minimization in (4) has an implicit restriction: $\mathbf{x}^{i}$ must be reachable from $\mathrm{x}^{i-1}$, otherwise $\Delta$ is infinite. Rewrite (4) as

$$
\begin{array}{r}
V^{i}\left(t^{i-1}, \mathbf{x}^{i-1}\right)= \\
\inf \left\{t^{i}-t^{i-1}+\mathcal{K}^{i}\left(t^{i}, \boldsymbol{\xi}\left(t^{i} ; t^{i-1}, \mathbf{x}^{i-1}, \boldsymbol{u}\right)\right)\right. \\
\left.: t^{i} \geq t^{i-1}, \boldsymbol{u} \in \mathcal{U}_{t^{i-1}}^{t^{i}}\right\},
\end{array}
$$

where $\mathcal{U}_{s}^{t}$ is the set of measurable controls $\boldsymbol{u}:[s, t] \rightarrow \mathbf{R}^{2}$ which satisfy $\|\boldsymbol{u}(\tau)\| \leq r$ for almost all $\tau \in[s, t]$ and $\boldsymbol{\xi}(t ; s, \mathbf{x}, \boldsymbol{u})$ is the value at time $t$ of a trajectory of (1) satisfying $\boldsymbol{\xi}(s ; s, \mathbf{x}, \boldsymbol{u})=\mathbf{x}$. Thus, finding $\left(t^{i}, \mathbf{x}^{i}\right)$ is equivalent to solving an optimal stopping problem with boundary cost $\mathcal{K}^{i}$. The application of the DPP to this problem gives

$$
\begin{aligned}
0=\max & \left\{V^{i}(t, \mathbf{x})-\mathcal{K}^{i}(t, \mathbf{x}),\right. \\
- & \left.1+r\left\|\nabla_{\mathbf{x}} V^{i}\right\|-\frac{\partial V^{i}}{\partial t}-\nabla_{\mathbf{x}} V^{i} \cdot \boldsymbol{v}(t, \mathbf{x})\right\},
\end{aligned}
$$

and the optimal control is given in feedback form as

$$
\boldsymbol{u}(t, \mathbf{x})=-r \frac{\nabla_{\mathbf{x}} V^{i}(t, \mathbf{x})}{\left\|\nabla_{\mathbf{x}} V^{i}(t, \mathbf{x})\right\|}
$$

Equation (6) is a variational inequality which expresses the intuitive fact that at each point the optimal decision is either to deploy the next AUV, in which case the value function equals the boundary cost at that point, or to move along an optimal trajectory, in which case the derivative of the value function along the trajectory is equal to -1 .

Bardi and Capuzzo-Dolcetta [3] give a derivation of (6) for the time-invariant discounted-cost case which is easily adapted to the problem at hand. The derivation requires the technical condition that $\mathcal{K}^{i}$ be uniformly continuous for each $i$, which holds in this case assuming that the $\theta^{i}$ are bounded and uniformly continuous.

Lemma 2: Assume that $\boldsymbol{v}$ in (1) is globally Lipschitz in $(t, \mathbf{x})$. If $\mathcal{K}^{i}$ is bounded and uniformly continuous, then so is $V^{i}$ (as defined by (5)).

Proof: Consider the control system with dynamics

$$
\dot{z}=g(z, \boldsymbol{u})=(1, \boldsymbol{u}+\boldsymbol{v}(z))
$$

where $z=(t, \mathbf{x}) \in \mathbf{R} \times \mathbf{R}^{2}$. Let $\boldsymbol{\zeta}(\tau ; z, \boldsymbol{u})$ denote the trajectory of this system satisfying $\boldsymbol{\zeta}(0 ; z, \boldsymbol{u})=z$. Setting

$$
\begin{aligned}
& \vartheta(z)=V^{i}(t, \boldsymbol{x}) \\
& k(z)=\mathcal{K}^{i}(t, \boldsymbol{x}),
\end{aligned}
$$

we have

$$
\vartheta(z)=\inf _{\tau \geq 0, \boldsymbol{u} \in \mathcal{U}}\{\tau+k(\boldsymbol{\zeta}(\tau ; z, \boldsymbol{u}))\},
$$

where $\mathcal{U}$ is the set of measurable $\boldsymbol{u}: \mathbf{R}_{\geq 0} \rightarrow \mathbf{R}^{2}$ which satisfy $\|\boldsymbol{u}(t)\| \leq r$ almost everywhere. This means $\vartheta$ is the value function for a time-invariant optimal stopping problem.

Let $M_{k}$ be such that $0 \leq k(z) \leq M_{k}$ for all $z$. Since a feasible solution to the optimization problem (8) is $\tau=0$ and $\boldsymbol{u}$ arbitrary, it follows that

$$
V(z) \leq k(z) \leq M_{k}
$$

for each $z$, so $\vartheta$ is bounded. Additionally, since for $\tau>M_{k}$ and any $\boldsymbol{u}$ we have

$$
\tau+k(\boldsymbol{\zeta}(\tau ; z, \boldsymbol{u}))>M_{k}
$$

equation (8) can be rewritten as

$$
\vartheta(z)=\inf _{0 \leq \tau \leq M_{k}, \boldsymbol{u} \in \mathcal{U}}\{\tau+k(\boldsymbol{\zeta}(\tau ; z, \boldsymbol{u}))\} .
$$

Fix $z_{0}$ and $\varepsilon>0$ and pick $\tau \in\left[0, M_{k}\right]$ and $\boldsymbol{u} \in \mathcal{U}$ so that

$$
\vartheta\left(z_{0}\right) \geq \tau+k\left(\boldsymbol{\zeta}\left(\tau ; z_{0}, \boldsymbol{u}\right)\right)-\varepsilon .
$$

For any $z$ we have

$$
\vartheta(z) \leq \tau+k\left(\boldsymbol{\zeta}\left(\tau ; z_{0}, \boldsymbol{u}\right)\right)
$$

so that

$$
\begin{aligned}
\vartheta(z)-\vartheta\left(z_{0}\right) & \leq k(\boldsymbol{\zeta}(\tau ; z, \boldsymbol{u}))-k\left(\boldsymbol{\zeta}\left(\tau ; z_{0}, \boldsymbol{u}\right)\right)+\varepsilon \\
& \leq\left|k(\boldsymbol{\zeta}(\tau ; z, \boldsymbol{u}))-k\left(\boldsymbol{\zeta}\left(\tau ; z_{0}, \boldsymbol{u}\right)\right)\right|+\varepsilon \\
& \leq \omega_{k}\left(\left\|\boldsymbol{\zeta}(\tau ; z, \boldsymbol{u})-\boldsymbol{\zeta}\left(\tau ; z_{0}, \boldsymbol{u}\right)\right\|\right)+\varepsilon
\end{aligned}
$$

where $\omega_{k}: \mathbf{R}_{\geq 0} \rightarrow \mathbf{R}_{\geq 0}$ is an increasing modulus of continuity for $k$ (existence of $\omega_{k}$ is implied by the uniform continuity of $k$ ). Letting $L_{g}>0$ be a global Lipschitz constant 
for $g$, we have a bound on the distance between the two trajectories [3]:

$$
\left\|\boldsymbol{\zeta}(\tau ; z, \boldsymbol{u})-\boldsymbol{\zeta}\left(\tau ; z_{0}, \boldsymbol{u}\right)\right\| \leq \exp \left(L_{g} \tau\right)\left\|z-z_{0}\right\|,
$$

so that

$$
\begin{aligned}
\vartheta(z)-\vartheta\left(z_{0}\right) & \leq \omega_{k}\left(\exp \left(L_{g} \tau\right)\left\|z-z_{0}\right\|\right)+\varepsilon \\
& \leq \omega_{k}\left(\exp \left(L_{g} M_{k}\right)\left\|z-z_{0}\right\|\right)+\varepsilon
\end{aligned}
$$

from which, since the bound depends only on $\left\|z-z_{0}\right\|$,

$$
\left|\vartheta(z)-\vartheta\left(z_{0}\right)\right| \leq \omega_{k}\left(\exp \left(L_{g} M_{k}\right)\left\|z-z_{0}\right\|\right)+\varepsilon
$$

and thus $\vartheta$ is uniformly continuous.

Hence, if $\theta^{n}$ is bounded and uniformly continuous, then so is $V^{n}$, and by induction, if each $\theta^{i}$ is bounded and uniformly continuous then all the $V^{i}$ are bounded and uniformly continuous, so that all the $\mathcal{K}^{i}$ are too.

Equation (6) obviously requires that $V^{i}$ be differentiable at $(t, \mathbf{x})$, which is not necessarily the case. Solutions of (6) are typically defined in a generalized (viscosity) sense [3]. Thus, in absolute rigor it would be necessary to prove that (5) gives the unique viscosity solution of (6), but we do not provide such a proof in this paper. A proof for the discounted cost case is given in Bardi and Capuzzo-Dolcetta [3].

Given the carrier deployment time and position $\left(t^{0}, \mathbf{x}^{0}\right)$ the optimal solution for $i=1, \ldots, n$ comes from integrating

$$
\dot{\mathbf{x}}(t)=-r \frac{\nabla_{\mathbf{x}} V^{i}(t, \mathbf{x}(t))}{\left\|\nabla_{\mathbf{x}} V^{i}(t, \mathbf{x}(t))\right\|}+\boldsymbol{v}(t, \mathbf{x}(t))
$$

with the initial condition $\mathbf{x}\left(t^{i-1}\right)=\mathbf{x}^{i-1}$, until the solution reaches the set $\left\{(t, \mathbf{x}): V^{i}(t, \mathbf{x})=\mathcal{K}^{i}(t, \mathbf{x})\right\}$ (by the proof of Lemma 2, every trajectory of (9) reaches this set in finite time). If this set is reached at time $\tau$, then set $t^{i}=\tau$ and $\mathbf{x}^{i}=\mathbf{x}(\tau)$, increment $i$ and repeat.

\section{B. Constraints}

In the above we have not considered constraints on the deployment positions and times $\left(t^{i}, \mathbf{x}^{i}\right)$. Let $M \geq 0$ be such that if $V^{1}(t, \mathbf{x}) \geq M$; then it is not feasible to deploy the carrier from $\mathbf{x}$ at time $t$ ( $M$ can be derived from the maximum mission duration). If there are constraints on the AUVs' deployment locations, i.e. AUV $i$ may only be deployed at $(t, \mathbf{x}) \in \Gamma^{i}$, then one modifies $\theta^{i}$ so that $\theta^{i}(t, \mathbf{x})=M$ for $(t, \mathbf{x}) \notin \Gamma^{i}$ and $\theta^{i}(t, \mathbf{x})$ is unchanged away from the complement of $\Gamma^{i}$. This must be done so that $\theta^{i}$ remains uniformly continuous, resulting in a conservative approximation of the constraint set.

\section{Numerical computation}

Typically, variational inequalities of the form (6) do not have a closed-form solution, so numerical methods must be used to approximate the solution. Most numerical methods will compute the solution over a discrete grid on a hyperrectangle in the $(t, \mathbf{x})$-space. In our problem this is

$$
D=[\underline{t}, \bar{t}] \times\left[\underline{x}_{1}, \bar{x}_{1}\right] \times\left[\underline{x}_{2}, \bar{x}_{2}\right] .
$$

Note that the dimension of the space in which the solutions are computed is independent of the number of AUVs. The gradient $\nabla_{\mathbf{x}} V^{i}$ in (7) can be approximated at the grid points via a finite difference approximation and interpolated to points of $D$ not in the grid. The ordinary differential equation (9) will then be numerically solved using any integration method. Naturally, the stopping condition $V^{i}=\mathcal{K}^{i}$ must be relaxed to $V^{i}+\varepsilon>\mathcal{K}^{i}$ for some appropriate tolerance parameter $\varepsilon$.

In the examples below we use our open-source parallel implementation $^{1}$ of the fast sweeping method (FSM) [13, $9,1]$ to compute an approximate solution of (6). The FSM is an iterative method which can be combined with several different discretization methods. Our implementation uses a Lax-Friedrichs type discretization described in Kao et al. [9].

The FSM is typically applied to partial differential equations, so in what follows we show how it can be applied to the variational inequality in question. Writing

$$
H(z, p)=r\left\|\left(p_{2}, p_{3}\right)\right\|-p_{1}-\left(p_{2}, p_{3}\right) \cdot \boldsymbol{v}(z)-1,
$$

where $z=(t, \mathbf{x})$ and $p=\left(p_{1}, p_{2}, p_{3}\right) \in \mathbf{R}^{3},(6)$ is written as

$$
0=\max \left\{V^{i}(z)-\mathcal{K}^{i}(z), H\left(z, \nabla V^{i}(z)\right)\right\} .
$$

Kao et al. [9] show that the Lax-Friedrichs discretization of $H\left(z, \nabla V^{i}(z)\right)$ results in an expression of the form

$$
\alpha(z) \hat{V}^{i}(z)-\hat{H}\left(z,\left\{\hat{V}^{i}(y)\right\}_{y \in \mathcal{N}(z)}\right)
$$

for each gridpoint $z$, where $\alpha(z)>0, \hat{V}^{i}$ denotes the numerical approximation of $V^{i}$ at the gridpoints, and $\mathcal{N}(z)$ is the set of grid neighbors of $z$. An explicit expression for $\alpha$ is given in Kao et al. [9]. Hence (6) is discretized as

$$
\begin{aligned}
0=\max \left\{\hat{V}^{i}(z)-\mathcal{K}^{i}(z)\right. & \\
& \left.\alpha(z) \hat{V}^{i}(z)-\hat{H}\left(z,\left\{\hat{V}^{i}(y)\right\}_{y \in \mathcal{N}(z)}\right)\right\}
\end{aligned}
$$

or equivalently, since $\alpha$ is positive,

$$
\begin{aligned}
& 0=\max \left\{\alpha(z)\left(\hat{V}^{i}(z)-\mathcal{K}^{i}(z)\right)\right. \\
&\left.\alpha(z) \hat{V}^{i}(z)-\hat{H}\left(z,\left\{\hat{V}^{i}(y)\right\}_{y \in \mathcal{N}(z)}\right)\right\}
\end{aligned}
$$

and this can be solved for $\hat{V}^{i}(z)$ to give

$$
\hat{V}^{i}(z)=\min \left\{\mathcal{K}^{i}(z), \alpha(z)^{-1} \hat{H}\left(z,\left\{\hat{V}^{i}(y)\right\}_{y \in \mathcal{N}(z)}\right)\right\} .
$$

The second branch of the min is the standard update formula of the FSM (see Kao et al. [9] for details).

\section{NUMERICAL EXAMPLES}

We now illustrate our approach with two numerical examples. The AUVs are assumed to have dynamics identical to the carrier, with the norm of the control signal of AUV $i$ bounded by $r^{i}$. The task of AUV $i$ is to reach some point $\mathbf{x}_{T}^{i} \in \mathbf{R}^{2}$ in minimal time. Hence $\theta^{i}$ is the value function of

\footnotetext{
${ }^{1}$ https://github.com/mcpca/marlin
} 
a minimum time control problem, and we also compute it using the FSM.

The following holds in the two examples: i) The value functions $V^{i}$ will be computed over the set $(t, \mathbf{x}) \in[0,4] \times$ $[-1,1] \times[-1,1]$; ii) The grid over which the value functions are computed has resolutions of, respectively, 0.01 and 0.015 in the temporal and spatial dimensions; iii) The deployment time and position AUV $i$ is constrained to lie on the set $\Gamma^{i}=$ $\left\{(t, \mathbf{x}): \theta^{i}(t, \mathbf{x}) \leq 0.7\right.$ and $\left.x_{2} \leq 0.5\right\}$; and, iv) For AUV $i$, $r=1.5$ and $r^{i}=1.0$.

The constraint $\theta^{i}(t, \mathbf{x}) \leq 0.7$ represents a fuel constraint, which is translated into a task duration constraint. This can be done because the expended power is proportional to the cube of the magnitude of the control. Since the AUVs will travel at maximum speed after deployment (because these are solving a minimal time optimal control problem), the total energy consumption of vehicle $i$ is proportional to $\theta^{i}$.

\section{A. Two AUVs and zero ocean currents}

Here, $n=2$ and $\boldsymbol{v}$ is identically zero. The target positions of the AUVs are $\mathbf{x}_{T}^{1}=(-0.8,0.8)$ and $\mathbf{x}_{T}^{2}=(0.8,0.8)$.

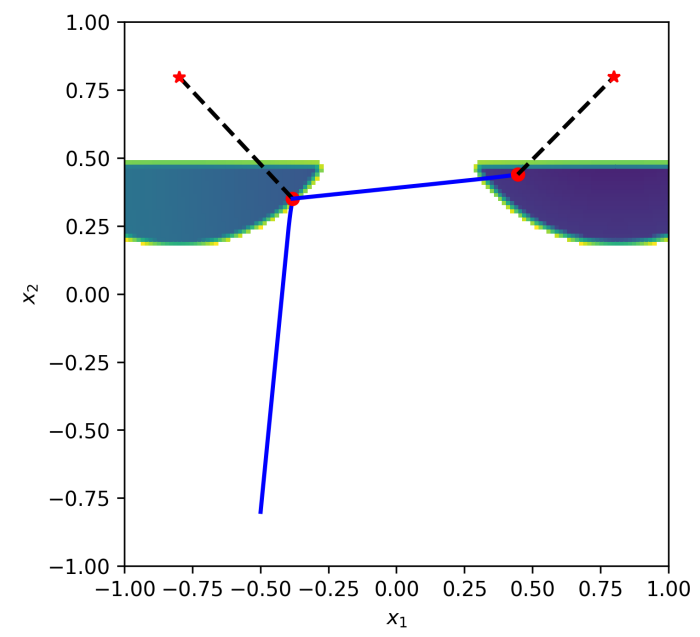

Fig. 1. Trajectory of the carrier and deployment positions of the AUVs.

Fig. 1 depicts the optimal solution for $t^{0}=0, \mathbf{x}^{0}=$ $(-0.5,-0.8)$. The carrier trajectory is plotted in blue and the deployment positions of the AUVs are indicated by the red circle. The red stars indicate the target positions of the AUVs, and the dashed lines the trajectories of the AUVs from the deployment to the target positions. The colored regions indicate the values of $\mathcal{K}^{i}$ on the constraint sets $\Gamma^{i}$ at the instant of time at which the corresponding AUV is deployed. Note that the values increase rapidly near the boundary of the sets (indicated by the lighter green color) due to the modification of $\theta^{i}$ according to the remark in IV-B. As expected, the trajectories of the carrier are straight lines, because the velocity of the current is zero, Moreover, the AUVs are expected to be deployed at the boundary of the respective constraint sets $\Gamma^{i}$. In fact, since the global mission time is dominated by $\mathcal{T}^{2}$, one expects the first and second AUVs to be deployed, respectively, at the boundary of the region $\left\{(t, \mathbf{x}): \theta^{i}(t, \mathbf{x}) \leq 0.7\right\}$, and near the region $x_{2}=0.5$ (the carrier is faster than the AUVs).

\section{B. Three AUVs and non-zero ocean currents}

Here, $n=3$ with target positions $\mathbf{x}_{T}^{1}=(-0.8,0.8), \mathbf{x}_{T}^{2}=$ $(0.0,0.8)$ and $\mathbf{x}_{T}^{3}=(0.8,0.8)$. The vector field $\boldsymbol{v}$ is given by

$$
\boldsymbol{v}(t, \mathbf{x})=\left(\begin{array}{c}
b-A \sin \left(\pi x_{1}-2 \pi f t\right) \cos \pi x_{2} \\
A \cos \left(\pi x_{1}-2 \pi f t\right) \sin \pi x_{2}
\end{array}\right)
$$

with $b=0.5, A=0.4, f=1.0$ (see Harrison et al. [8] for the motivation to use this class of vector fields).

Fig. 2 shows the optimal solutions for two different values of $t^{0}$ with $\mathbf{x}^{0}=(0.0,-0.8)$ in both cases. Vectors representing the velocity of the current are depicted along the carrier and AUV trajectories in cyan and green colors, respectively. Note that for $t^{0}=0.4$ the sets $\Gamma^{1}$ and $\Gamma^{2}$ intersect. In this particular example the two AUVs are deployed at the same time.

\section{CONCLUSIONS AND FUTURE WORK}

In this paper we presented a solution approach for the problem of deploying a set of vehicles in order to minimize the global mission time. A concise formulation of the problem in the framework of dynamic optimization was provided, followed by a solution approach based on the dynamic programming principle. The resulting variational inequalities are solved numerically to find the solution in the form of a set of value functions. This computationally expensive operation is efficiently performed by our parallel implementation of a solver [1]. Given the desired time and location of departure of the carrier, those value functions can then be used to determine approximations of the optimal trajectory for this vehicle, along with the deployment times and locations for all AUVs.

Future research directions will include the solution of problems where the deployment sequence is not given in advance and the investigation of the effect of non-zero AUV deployment times on the solution of the problem.

\section{ACKNOWLEDGMENTS}

This paper reports work partially supported by the following projects: "Marine robotics research infrastructure network - EUMR" funded by the EU Horizon 2020 programme under grant agreement No. 731103; "Sistema de Gestão de Operações com base em Veículos Robóticos Inteligentes para a Exploração do Mar Global a partir de Portugal - Oceantech" approved through the Incentive Scheme R\&TD Co-promotion Projects and co-funded by the European Regional Development Fund (ERDF), supported by Portugal2020 through Compete2020 (ref POCI-01-0247FEDER-024508); "European Multidisciplinary Seafloor and Water Column Observatory-Portugal - EMSO-PT' funded by the ERDF through Compete2020 and by FCT (ref. PINFRA / 22157/2016 - POCI-01-0145-FEDER-022157); and, "Sistema baseado em veículos autónomos para observação oceanográfica de longa duração - ENDURANCE", funded by NORTE2020 under the Portugal2020 Partnership Agreement through ERDF (ref. 17804). 


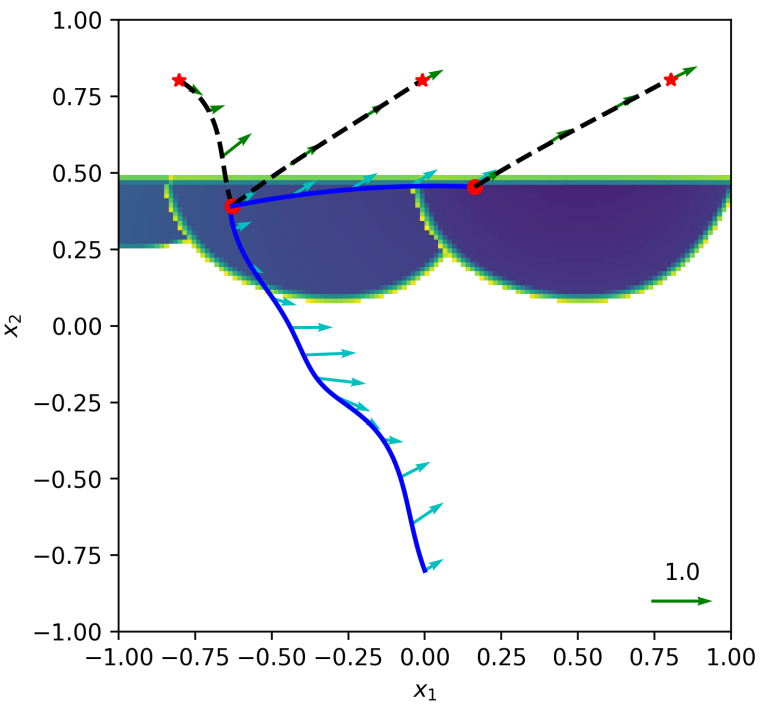

(a) $t^{0}=0.4$

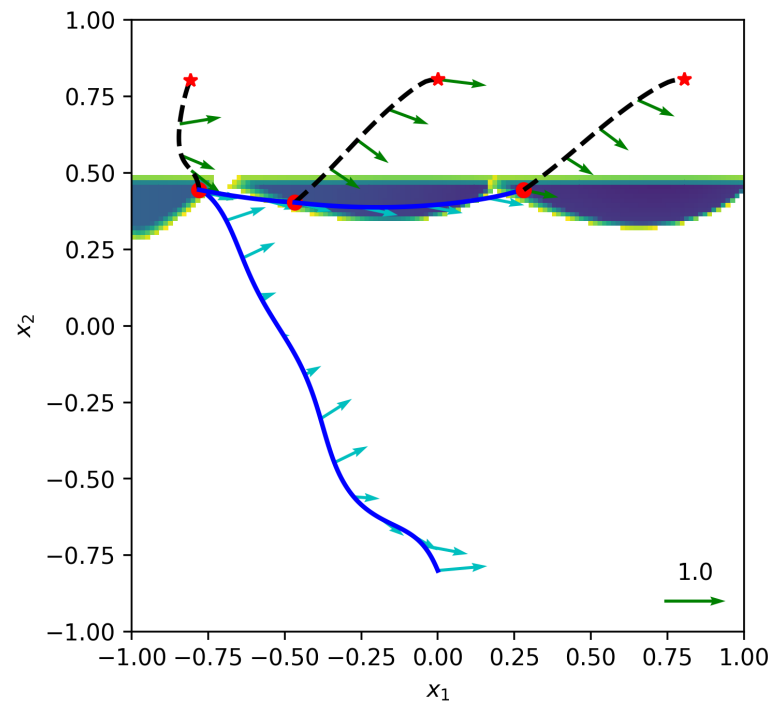

(b) $t^{0}=0.7$

Fig. 2. Optimal solutions for different values of $t^{0}$

\section{REFERENCES}

[1] Miguel Aguiar et al. "Optimizing autonomous underwater vehicle routes with the aid of high resolution ocean models". In: OCEANS 2019 MTS/IEEE SEATTLE. IEEE, Oct. 2019. DOI: 10.23919 / oceans 40490 . 2019.8962569.

[2] Ken Alton and Ian Mitchell. "Efficient dynamic programming for optimal multi-location robot rendezvous". In: Proceedings of the IEEE Conference on Decision and Control. Jan. 2009, pp. 2794-2799.

[3] Martino Bardi and Italo Capuzzo-Dolcetta. Optimal Control and Viscosity Solutions of Hamilton-JacobiBellman Equations. Birkhäuser Boston, 1997. DOI: 10.1007/978-0-8176-4755-1.

[4] J. Borges de Sousa and J. Estrela da Silva. "Optimal path coordination problems". In: 2008 47th IEEE Conference on Decision and Control. 2008, pp. 3113-3118.

[5] J. Borges de Sousa et al. "A verified hierarchical control architecture for co-ordinated multi-vehicle operations". In: International Journal of Adaptive Control and Signal Processing 21.2-3 (2007), pp. 159-188.

[6] Michael Branicky and Sanjoy Mitter. "Algorithms for optimal hybrid control". In: Proceedings of the 34th IEEE Conference on Decision and Control. Vol. 3. Jan. 1996, 2661-2666 vol.3. ISBN: 0-7803-2685-7. DOI: $10.1109 / \mathrm{CDC} .1995 .478514$.

[7] Jonathan C. Las Fargeas, Pierre T. Kabamba, and Anouck R. Girard. "Path planning for information acquisition and evasion using marsupial vehicles". In: American Control Conference, ACC 2015, Chicago, IL, USA, July 1-3, 2015. IEEE, 2015, pp. 3734-3739. DOI: $10.1109 /$ ACC.2015.7171910.

[8] Cheryl S. Harrison and Gary A. Glatzmaier. "Lagrangian coherent structures in the California Current
System - sensitivities and limitations". In: Geophysical \& Astrophysical Fluid Dynamics 106.1 (Feb. 2012), pp. 22-44.

[9] Chiu Yen Kao, Stanley Osher, and Jianliang Qian. "Lax-Friedrichs sweeping scheme for static Hamilton-Jacobi equations". In: Journal of Computational Physics 196.1 (May 2004), pp. 367-391. DOI: 10 . $1016 / j \cdot j c p .2003 .11 .007$.

[10] N. N. Krasovskii and A. I. Subbotin. Game-theoretical control problems. Springer Series in Soviet Mathematics. Springer-Verlag, 1988.

[11] Ali Pakniyat and Peter E. Caines. "On the Minimum Principle and Dynamic Programming for Hybrid Systems”. In: IFAC Proceedings Volumes 47.3 (2014). 19th IFAC World Congress, pp. 9629-9634.

[12] James A. Sethian and Alexander Vladimirsky. "Ordered Upwind Methods for Hybrid Control”. In: Hybrid Systems: Computation and Control. Ed. by Claire J. Tomlin and Mark R. Greenstreet. Berlin, Heidelberg: Springer Berlin Heidelberg, 2002, pp. 393-406.

[13] Hongkai Zhao. "A fast sweeping method for Eikonal equations". In: Mathematics of Computation 74.250 (May 2004), pp. 603-628. DOI: 10 . 1090/s $0025-$ 5718-04-01678-3. 